\title{
BEHAVIOUR OF THE VELOCITY OF ONE-DIMENSIONAL FLOWS IN POROUS MEDIA
}

\section{BY}

JUAN L. VAZQUEZ ${ }^{1}$

\begin{abstract}
The one-dimensional flow of gas of density $u$ through a porous medium obeys the equation $u_{t}=\left(u^{m}\right)_{x x}$, where $m>1, x \in \mathbf{R}$ and $t>0$. We prove that the local velocity of the gas, given by $v=-m u^{m-2} u_{x}$, not only is bounded for $t \geqslant \tau>0$ but approaches an $N$-wave profile as $t \rightarrow \infty$. $N$-waves are the typical asymptotic profiles for some first-order conservation laws, a class of nonlinear hyperbolic equations. The case $m \leqslant 1$ is also studied: there are solutions with unbounded velocity while others have bounded velocity.
\end{abstract}

0. Introduction. We consider the Cauchy problem for the one-dimensional porous media equation

$$
\begin{gathered}
u_{t}=\left(u^{m}\right)_{x x} \text { for } x \in \mathbf{R}, t>0, \\
u(x, 0)=u_{0}(x) \quad \text { for } x \in \mathbf{R},
\end{gathered}
$$

where $m$ is a positive constant and $u_{0}$ satisfies

$$
u_{0} \in L^{1}(\mathbf{R}), \quad u_{0} \geqslant 0 \text { a.e., } \quad u_{0} \neq 0 .
$$

Apart from its mathematical interest as a simple model of nonlinear evolution equation of (possibly degenerate or singular) parabolic type, equation (0.1) and its $N$-dimensional analogue appear in a number of applications; cf. [18] for a reference. In the typical application to the flow of gas through a porous medium we have $m>1$ and $u$ stands for the density of the gas. Writing $(0.1)$ as a conservation law for the mass

$$
u_{t}+(u \cdot v)_{x}=0
$$

gives for the local velocity of the gas the expression $v=-m u^{m-2} u_{x}$. We define the pressure as the potential of the velocity field, i.e. $v=-p_{x}$, so that

$$
p=(m /(m-1)) u^{m-1} \text {. }
$$

Received by the editors April 1, 1983 and, in revised form, December 1, 1983. The contents of this paper have been communicated to the 799th Meeting of the Amer. Math. Society, held in East Lansing, Michigan, on November 12, 1982.

1980 Mathematics Subject Classification. Primary 35B40, 35K55, 76S05.

Key words and phrases. Velocity of propagation, $N$-waves, flows in porous media, asymptotic behaviour, locally bounded variation.

${ }^{1}$ Supported in part by a Fulbright Award 1982-83. 
$M=\int u_{0}(x) d x$ is the total mass. We retain this terminology even if $m \leqslant 1$. If $m=1$ we recover the classical heat equation; the pressure is then $p=\lg u$. For $0<m<1$ the equation appears in plasma physics (cf. [6], where actually the three-dimensional equation is considered).

There is a family of model solutions corresponding to an initial mass concentrated at 0 , i.e. $u(x, 0)=M \delta(x), \delta$ being Dirac's delta function. They are given by the selfsimilar functions (cf. [3])

$$
\bar{u}(x, t ; M)= \begin{cases}t^{-1 /(m+1)}\left[A^{2}-\frac{m-1}{2 m(m+1)} \cdot \frac{x^{2}}{t^{2 /(m+1)}}\right]_{+}^{1 /(m-1)} & \text { if } m \neq 1, \\ A t^{-1 / 2} \exp \left(-x^{2} /(4 t)\right) & \text { if } m=1,\end{cases}
$$

where $A=a_{m} M^{(m-1) /(m+1)}$ for a certain constant $a_{m}>0$ if $m \neq 1, A=a_{1} M$ if $m=1 ;[\cdot]_{+}$means $\max (\cdot, 0)$. Since $(0.1)$ is invariant under $x$ - and $t$-translations, the functions $\bar{u}\left(x-x_{0}, t+\tau ; M\right)$ are also solutions of $(0.1)$ in $Q=\mathbf{R} \times(0, \infty)$ for every $x_{0} \in \mathbf{R}, \tau \geqslant 0$.

This paper is concerned with the behaviour of the velocity of the solutions of (0.1)-(0.3). In this respect, if we define an $N$-wave in the domain $Q_{T}=\mathbf{R} \times(0, T)$, $T>0$, as a function of the form

$$
N(x, t)= \begin{cases}c(t)(x-x(t)) & \text { if } r_{1}(t)<x<r_{2}(t) \\ 0 & \text { otherwise }\end{cases}
$$

for some functions $c(t), x(t), r_{1}(t), r_{2}(t)$ such that $c(t) \in C^{1}(0, T)$ and $r_{1}(t) \leqslant x(t)$ $\leqslant r_{2}(t)$, it is not difficult to see that for $m>1$ there are two kinds of solutions of (0.1) whose velocities are $N$-waves: first the model solutions $\bar{u}\left(x-x_{0}, t+\tau ; M\right)$, and in this case $T=\infty, c(t)=((m+1)(t+\tau))^{-1}, x(t)=x_{0}$ and $x_{0}-r_{1}(t)=r_{2}(t)$ $-x_{0}=r_{M}(t)$ defined in (0.8). Setting, without essential restriction, $x_{0}=\tau=0$ we obtain the one-parameter family of symmetric $N$-waves

$$
\bar{v}(x, t ; M)= \begin{cases}\frac{x}{(m+1) t} & \text { if }|x|<r_{M}(t) \\ 0 & \text { if }|x|>r_{M}(t)\end{cases}
$$

where

$$
r(t)=r_{M}(t)=c_{m} M^{(m-1) /(m+1)} t^{1 /(m+1)}, \quad c_{m}=a_{m}\left(\frac{2 m(m+1)}{m-1}\right)^{1 / 2}, \quad m>1 .
$$

Remark that $\bar{v}(x, t ; M)$ is positive in $\bar{\Omega}_{M}=\left\{(x, t):|x|<r_{M}(t)\right\}$.

$N$-waves can also be obtained from the following solutions (and their translates):

$$
\hat{u}(x, t ; T, C)=(T-t)^{-1 /(m+1)}\left[\frac{m-1}{2 m(m+1)} \cdot \frac{x^{2}}{(T-t)^{2 /(m+1)}}-C\right]_{+}^{1 /(m-1)}
$$

defined in $Q_{T} ; T>0$ and $C$ are arbitrary constants. For $C \geqslant 0$ we have also the solutions $\hat{u}_{+}(x, t ; T, C)=\hat{u}(x, t ; T, C) \cdot H(x)$ and $\hat{u}_{-}(x, t ; T, C)=$ $\hat{u}(x, t ; T, C) H(-x)$, where $H(x)=1$ if $x>0, H(x)=0$ if $x \leqslant 0$. All these solutions 
have $N$-wave velocities with $c(t)=((m+1)(t-T))^{-1}<0$ and correspond to nonintegrable initial data that cause a blow-up in finite time $T$. In fact, they serve as a model for the solutions of $(0.1)$ that blow up in finite time, cf. $[5,21]$ for results about this class of solutions, but we shall not deal with them here.

The main result of this paper consists in proving that for every solution of (0.1)-(0.3) with $m>1$ the velocity behaves asymptotically as $t \rightarrow \infty$ like the $N$-wave (0.7) where $M=\int u_{0}(x) d x$. Therefore, there is essentially a one-parameter family of asymptotic profiles. The convergence results are precisely stated and proved in $\$ \S 1$ and 2. After introducing a rescaling of the variables $v$ and $x$ we prove that $v(\cdot, t) \rightarrow \bar{v}(\cdot, t)$ as $t \rightarrow \infty$ in the sense of graphs. Since $\bar{v}$ is discontinuous we cannot expect to have uniform convergence in the usual sense.

It is remarkable that this asymptotical $N$-wave behaviour occurs in several first order equations of hyperbolic type that develop singularities in the form of shock waves, like the single conservation law with a convex nonlinearity

$$
u_{t}+\left(|u|^{n}\right)_{x}=0, \quad n>1,(x, t) \in Q .
$$

and the one with an odd nonlinearity

$$
u_{t}+\left(|u|^{n-1} u\right)_{x}=0, \quad n>1,(x, t) \in Q .
$$

Both equations admit $N$-wave solutions for their respective velocities. Thus if we set $v=|u|^{n-1} \operatorname{sign}(u)$ in $(0.10)$ we have a $t w o$-parameter family of solutions of $(0.10)$ given in terms of $v$ by

$$
\bar{v}(x, t ; p, q)= \begin{cases}x / n t & \text { if }-p t^{1 / n}<x<q t^{1 / n} \\ 0 & \text { if } x>q t^{1 / n} \text { or } x<-p t^{1 / n}\end{cases}
$$

where $p, q \geqslant 0$. The corresponding solution $\hat{u}(x, t ; p, q)$ solves $(0.10)$ with initial condition $u(x, 0)=M \delta(x)$ where

$$
M=\frac{n-1}{n}\left(q^{n /(n-1)}-p^{n /(n-1)}\right) .
$$

For equation $(0.11)$ we set $v=|u|^{n-1}$ and obtain the $N$-waves $\bar{v}(x, t ; 0, q)$, with $|M|=((n-1) / n) q^{n /(n-1)}$ and then $\hat{u}(x, t ; M)=\bar{v}(x, t ; 0, q)^{1 /(n-1)} \cdot \operatorname{sign}(M)$. In this case we have a one-parameter family.

It has been known for a long time that $N$-waves represent the asymptotic profiles of conservation laws of the type $u_{t}+f(u)_{x}=0$ under different restrictions on $u(x, 0)$ and $f$ (cf. Lax [14], Di Perna [10], Dafermos [9], Liu [15]). Liu and Pierre's paper [16] contains a theory of existence and uniqueness for the Cauchy problems $(0.10),(0.2)$ and $(0.11),(0.2)$, as well as a study of the asymptotics: For every $u_{0} \in L^{1}(\mathbf{R})$ there are $p, q$ satisfying $(0.13)$ such that the solution of $(0.10),(0.2)$ approaches as $t \rightarrow \infty$ the selfsimilar solution $\hat{u}(x, t ; p, q)$. In the case of the problem $(0.11),(0.2)$ the convergence is towards $\hat{u}(x, t ; M)$.

These convergences take place in $L^{1}(\mathbf{R})$. Convergence in uniform graph-norm can be found however in [14] (for $f$ such that $f^{\prime \prime}>0$ and $u_{0}$ with compact support).

Close similarity with $(0.7)$ is obtained in the preceding results by setting $n=m+1$. But in order to have an analogous definition for the velocities it is convenient to 
integrate with respect to $x$ our conservation laws, for instance $(0.10)$. We obtain the equation

$$
w_{t}+\left|w_{x}\right|^{n}=0, \quad n>1,(x, t) \in Q,
$$

where $w(x, t)=\int_{-\infty}^{x} u(s, t) d s$. The Cauchy problem for a class of equations including (0.14) has been studied by Conway and Hopf [8]. Writing (0.14) as $w_{t}+v w_{x}=0$ gives, for the velocity of the solutions of $(0.14)$, the value $v=\left|w_{x}\right|^{n-2} w_{x}\left(=|u|^{n-2} u\right)$. Also $u_{0} \in L^{1}(\mathbf{R})$ means $w(x, 0) \in L^{\infty}(\mathbf{R})$. If we also impose on $w(x, 0)$ the condition that it vanish as $|x| \rightarrow \infty$, the family of admissible $N$-waves for $v$ reduces to the symmetric ones, $p=q$, and we obtain a complete analogy between the asymptotics of this problem and that of problem $(0.1)-(0.3)$.

In case $m \leqslant 1$ equation ( 0.1$)$ admits only one "infinite $N$-wave" defined in $Q$ by $x /((m+1) t)$. It represents the velocity of the model solutions $(0.5)$ and it is an unbounded function of $x$ for every $x>0$. We prove that for every solution of $(0.1)-(0.3)$ this unique $N$-wave is the asymptotic profile of the velocity. However, the rates of convergence differ according to the initial data: thus if $u_{0}$ has compact support then the velocity cannot be a bounded function of $x$ for any $t>0$, a sharp difference with respect to the case $m>1$.

Nevertheless there are solutions of $(0.1)-(0.3)$ with bounded velocity in $Q$ even if $m \leqslant 1$. This is even true for any equation of the form

$$
u_{t}=(\varphi(u))_{x x}
$$

where $\varphi$ is a continuous, nondecreasing function $[0, \infty) \rightarrow[0, \infty)$. This finite-velocity property for $m \leqslant 1$ seems to have been unnoticed in the literature. Let us remark that an initial function $u_{0}$ such that $\left(u_{0}^{m-1}\right)_{x}$ is bounded can never vanish if $m \leqslant 1$.

The paper is organized as follows. $§ 1$ : statement of results if $m>1$; §2: proofs; §3: the case $m<1 ; \S 4$ : solutions with bounded velocity for $(0.15)$.

1. The case $m>1$. We consider here the problem $(0.1)-(0.3)$ for $m>1$. A unique solution $u$ exists for this problem and $u \in C\left([0, T]: L^{1}(\mathbf{R})\right) \cap L^{\infty}(\mathbf{R} \times(\delta, \infty))$ for every $\delta>0 ; u$ is nonnegative and it is a smooth classical solution of $(0.1)$ in the domain of dependence $\Omega=\{(x, t): u(x, t)>0\}$ where the equation is nondegenerate parabolic. However, at the transition between $\Omega$ and the "void" region where $u=0$, the velocity is in general discontinuous. Two important properties we shall need in the sequel are (i) the total mass is conserved, i.e. for every $t>0$

$$
\int u(x, t) d x=\int u_{0}(x) d x,
$$

and (ii) there is a lower bound for the (distributional) derivative $p_{x x}$ in $Q$ :

$$
p_{x x} \geqslant-1 /(m+1) t \text {. }
$$

Estimate (1.2) is due to Aronson and Bénilan [2]; cf. [19] for further details.

We are interested in comparing the solution $u(x, t)$ with the selfsimilar solution $\bar{u}(x, t ; M)$ having the same mass $M$. In this respect, Kamin [13] and Friedman and Kamin [11] have shown that the following convergence takes place uniformly in $x$ :

$$
t^{1 /(m+1)}|u(x, t)-\bar{u}(x, t ; M)| \rightarrow 0 \quad \text { as } t \rightarrow \infty .
$$


If $u_{0}$ has compact support, then, for every $t>0, u(\cdot, t)$ is compactly supported and there exist two monotone curves, $x=\zeta_{1}(t)$ and $x=\zeta_{2}(t)$, called free-boundaries or interfaces, that bound the domain of dependence to the left and right, respectively (with respect to an $(x, t)$-frame). We have shown in [19] that in this case a fairly complete description of the large-time behaviour can be done. In fact, if $u(x, t)$ is such a solution and $\bar{u}=\bar{u}(x-\bar{x}, t ; M)$ is the selfsimilar solution with same mass centered at the center of mass

$$
\bar{x}=M^{-1} \int x u_{0}(x) d x,
$$

then $u, p, v, \zeta_{i}$ and $\zeta_{i}^{\prime}$ converge to $\bar{u}, \bar{p}, \bar{v}, \bar{\zeta}_{i}$ and $\bar{\zeta}_{i}^{\prime}$ as $t \rightarrow \infty$ as follows:

$$
\begin{gathered}
(-1)\left(\zeta_{i}(t)-\bar{\zeta}_{i}(t)\right) \downarrow 0, \\
t\left|\zeta_{i}^{\prime}(t)-\bar{\zeta}_{i}^{\prime}(t)\right| \rightarrow 0, \\
t|v(x, t)-\bar{v}(x, t)| \rightarrow 0 \quad \text { uniformly in }|x| \leqslant r(t), \\
t^{m /(m+1)}|p(x, t)-\bar{p}(x, t)| \rightarrow 0 \quad \text { uniformly in } x \in \mathbf{R},
\end{gathered}
$$

where $\bar{\zeta}_{i}(t)=\bar{x}+(-1)^{i} r(t), r(t)$ defined in (0.6). From (1.8) an estimate for $u-\bar{u}$ follows which implies a rate of convergence faster than (1.3).

In this paper we establish precise results about the comparison of density, pressure and velocity with their model counterparts under the assumptions (0.3) on $u_{0}$. As $t$ grows large we obtain an accurate description of the behaviour of the solutions. Our first result is the following

THEOREM 1. Let $u(x, t)$ be a solution of $(0.1),(0.2)$ with initial data $u_{0}$ satisfying (0.3). Then for every $t>0$

$$
\begin{gathered}
u(x, t)<\max _{x \in \mathbf{R}} \bar{u}(x, t ; M)=d_{m} M^{2 /(m+1)} t^{-1 /(m+1)}, \quad x \in \mathbf{R}, \\
|v(x, t)|<\max _{x \in \mathbf{R}}|\bar{v}(x, t ; M)|=c_{m} M^{(m-1) /(m+1)} t^{-m /(m+1)}, \quad x \in \mathbf{R},
\end{gathered}
$$

where $M>0$ is the mass of $u$ and $c_{m}, d_{m}$ are positive constants depending only on $m$. Moreover, for every $\varepsilon>0$ there exists $t_{\varepsilon}>0$ such that if $t \geqslant t_{\varepsilon}$

$$
\begin{gathered}
|v(x, t)-\bar{v}(x, t)| t^{m /(m+1)} \leqslant \varepsilon \quad \text { if }|| x|-r(t)| \geqslant \varepsilon r(t), \\
v(x, t) t^{m /(m+1)} \cdot \operatorname{sign}(x) \geqslant-\varepsilon \quad \text { if }|| x|-r(t)|<\varepsilon r(t) .
\end{gathered}
$$

Let us discuss these results: (1.9) is true also in the $N$-dimensional case $u_{t}=\Delta u^{m}$ (cf. [20]). The fact that for any solution of (0.1)-(0.3) and any $t>0$ the velocity is bounded was proved by Aronson [1] (with some additional hypothesis on $u_{0}$ ). Using a scaling argument (by means of the biparametric group of transformations $u^{\prime}=k u$, $x^{\prime}=L^{-1} x, t^{\prime}=L^{-2} k^{-m+1} t$, with $k, L>0$ that leaves the equation invariant) it follows easily that the rate of decay must be $t^{-m /(m+1)}$ (1.10) above gives the sharp constant in this estimate since (1.11) implies that $\max _{x} v(x, t) t^{m /(m+1)} \rightarrow$ $c_{m} M^{(m-1) \wedge m+1)}$ as $t \rightarrow \infty$. 
In order to visualize the meaning of $(1.11),(1.12)$ we introduce the rescaled variables

$$
y=\frac{x}{r(t)}, \quad P=\frac{(m+1) t}{r(t)^{2}} p, \quad V=\frac{(m+1) t}{r(t)} v .
$$

We have $V=-\partial P / \partial y$. The model solution $\bar{p}(x, t ; M)$ is transformed into the unique invariant (i.e. independent of $t$ ) profile

$$
\bar{P}(y)=\frac{1}{2}\left[1-y^{2}\right]+
$$

and the velocity becomes the unit symmetric $N$-wave $\bar{V}(y)=y$ if $|y|<1, \bar{V}(y)=0$ if $|y|>1$. Let $\bar{\Gamma}$ be the graph of the function $\bar{V}$ in the $(V, y)$-plane completed with the vertical segments $\{-1\} \times[-1,0]$ and $\{1\} \times[0,1]$. Then $(1.11),(1.12)$ can be restated thus: the graph $\Gamma(t)$ of the rescaled velocity of a solution of $(0.1)-(0.3)$ converges as $t \rightarrow \infty$ towards $\bar{\Gamma}$ in the metric topology of $\mathbf{R}^{2}$ (given e.g. by the norm $\|(V, y)\|=|V|$ $+|y|)$.

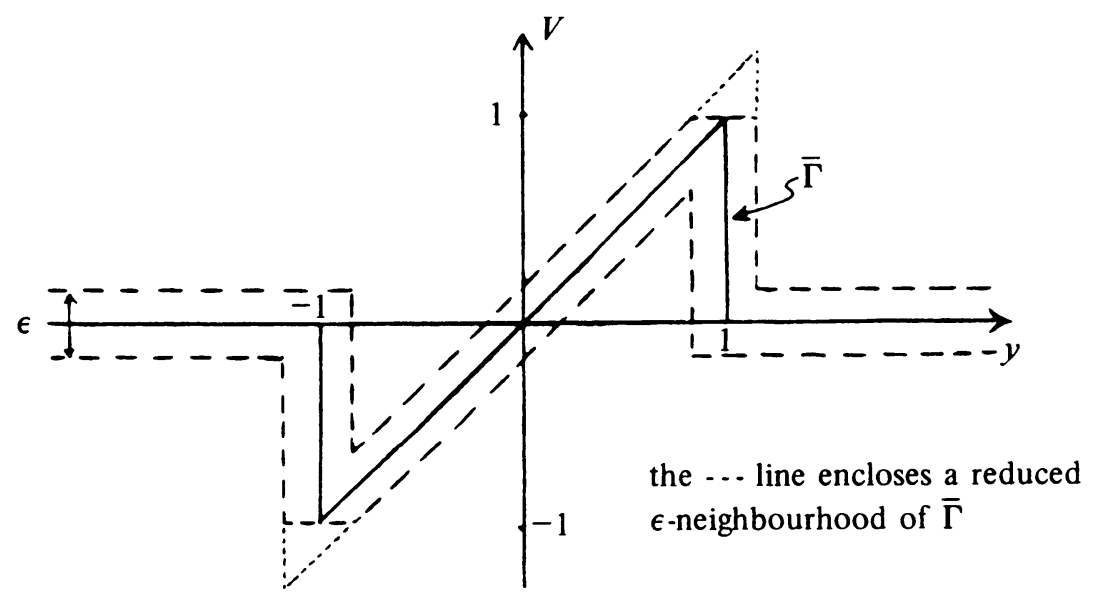

FIGURE 1

In fact, using (1.10) we can consider the "reduced" $\varepsilon$-neighborhoods shown in Figure 1.

In case $u_{0}$ has compact support (1.5) and (1.7) imply that the convergence in (1.11), (1.12) takes place with a rate $\varepsilon(t)=o\left(t^{-1 /(m+1)}\right)$. Nevertheless, for general $u_{0}$ the results of Theorem 1 are sharp as we show next.

THEOREM 2. For every function $\varphi:[0, \infty) \rightarrow[0, \infty)$ such that $\lim \varphi(t)=\infty$ as $t \rightarrow \infty$ there exists a continuous, nonnegative and integrable $u_{0}$ such that if $u(x, t)$ is the corresponding solution of $(0.1),(0.2)$ then

$$
\limsup _{t \rightarrow \infty}\left(\bar{u}(0, t ; M)-\max _{x} u(x, t)\right) \varphi(t) t^{1 /(m+1)}=\infty
$$




$$
\limsup _{t \rightarrow \infty}\left(\max _{x} \bar{v}(x, t ; M)-\max _{x} v(x, t)\right) \varphi(t) t^{m /(m+1)}=\infty,
$$

and we can choose $u_{0}$ symmetric, i.e. $u_{0}(x)=u_{0}(-x)$ for every $x$.

The $N$-wave $\bar{v}(x, t ; M)$ is a function of bounded variation in $x$ for every $t>0$, $v(\cdot, t) \in V B(\mathbf{R})$, and, in fact, if $\|\mid \cdot\|=\|\| \cdot \|_{\mathbf{R}}$ is the total variation of a function $\mathbf{R} \rightarrow \mathbf{R}$ we have

$$
\|\bar{v}(\cdot, t ; M)\|_{\mathbf{R}}=4 \max \bar{v}(\cdot, t ; M)=O\left(t^{-m \wedge m+1)}\right) .
$$

When we consider a generic solution of $(0.1)-(0.3)$ we can only assert that the velocity is a function of locally bounded variation. We have

THEOREM 3. Let $u(x, t)$ be a solution of $(0.1)-(0.3)$ and let $v(x, t)$ be its velocity. Then for any $t>0, v(\cdot, t) \in V B_{\text {loc }}(\mathbf{R})$. Moreover, for each $\varepsilon>0$ there exist $\delta=\delta_{\varepsilon}>0$ and $t_{\varepsilon}>0$ such that if $I_{\delta}(t)=\{x \in \mathbf{R}:|x| \leqslant(1+\delta) r(t)\}$ then for every $t>t_{\varepsilon}$

$$
\|v(\cdot, t)\|_{I_{\delta}(t)} \leqslant(1+\varepsilon)\|\bar{v}(\cdot, t ; M)\|_{\mathbf{R}} .
$$

If $u_{0}$ has compact support then $v(\cdot, t) \in V B(\mathbf{R})$ for all $t>0$ and

$$
\lim _{t \rightarrow \infty}\|v(\cdot, t)\| t^{m /(m+1)}=4 c_{m} M^{(m-1) /(m+1)}
$$

(1.19) is not true for every solution of $(0.1)-(0.3)$ : we exhibit an example of solution such that $\|v(\cdot, t)\|=\infty$ for every $t>0$. The function in the example has an infinite number of "humps" for every $t>0$.

Finally, we remark that the assumption $u_{0} \in L^{1}(\mathbf{R})$ is necessary in the above estimates, that lose their meaning if we let $M \rightarrow \infty$.

2. Proofs.

2.1. Proof of Theorem 1. Using rescaled variables and fixing $t>0$ we may assume that $P$ is a nonnegative function of $y \in \mathbf{R}$ such that its second derivative $P_{y y}$ satisfies $\boldsymbol{P}_{y y} \geqslant-1\left(\right.$ in $\left.\mathscr{D}^{\prime}(\mathbf{R})\right)$. Also $V=-P_{y}$ so that $V_{y} \leqslant 1$. Finally we have

$$
\int_{-\infty}^{\infty} P(y)^{1 /(m-1)} d y=\int_{-\infty}^{\infty} \bar{P}(y)^{1 /(m-1)} d y .
$$

Then (1.9), (1.10) follow from the next elementary

Lemma 2.1. Let $P, V$ be as above. Then $P(y) \leqslant 1 / 2$ and $|V(y)| \leqslant 1$. If equality holds for one of these estimates then up to translation of the $y$-axis $P=\bar{P}$ and $V=\bar{V}$.

Proof. Assume also that $P$ is smooth and bounded. If $P(y) \nless 1 / 2$ for every $y$ then $P$ has a maximum value $\geqslant 1 / 2$. This maximum is attained at some point (because of (2.1)); let this point be 0 . Then using $P_{y y} \geqslant-1$ we get

$$
P(y) \geqslant \bar{P}(y) \text { for }|y| \leqslant 1 \text {. }
$$

(2.2) is compatible with (2.1) only if $P=\bar{P}$.

As for $V$, if $V\left(y_{0}\right) \geqslant 1$ at some point $y_{0} \in \mathbf{R}$, let this point be 1 , since necessarily $P(1) \geqslant 0$ integrating $P_{y y} \geqslant-1$ twice from $y_{0}=1$ gives $P(y) \geqslant \bar{P}(y)$ for $-1 \leqslant y \leqslant 1$ and we conclude as above.

If $P$ is not smooth or bounded the result follows by approximation. 
END OF Proof of Theorem 1. To prove (1.11), (1.12) we can use a similar argument after writing the convergence (1.3) in the form

$$
\left|P(y, t)^{1 /(m-1)}-\bar{P}(y)^{1 /(m-1)}\right|<\varepsilon_{1}
$$

for all $t \geqslant t_{\varepsilon_{1}}$. We leave it to the reader to check that if $V(y, t)$ does not belong to the $\varepsilon$-neighborhood in Figure 1 with $\varepsilon=k \varepsilon_{1}$ for some constant $k=k(m)>0$, then double integration of $P_{y y} \geqslant-1$ allows us to arrive at a contradiction with (2.3).

2.2. Proof of TheOREM 2. Let $\left\{t_{n}\right\}$ be an increasing and divergent sequence such that $\varphi\left(t_{n}\right) \geqslant 3^{n}$. The function $u(x, t)$ that we seek will be the solution of $(0.1)$ with initial condition

$$
u_{0}(x)=\bar{u}(x, \tau ; 1-\varepsilon)+\sum_{i=1}^{\infty}\left(\bar{u}\left(x-x_{i}, \tau ; \varepsilon / 2^{i+1}\right)+\bar{u}\left(x+x_{i}, \tau ; \varepsilon / 2^{i+1}\right)\right)
$$

where $\tau>0$ and $0<\varepsilon<1 / 2$ are constants and $\left\{x_{i}\right\}$ is an increasing nonnegative sequence to be chosen conveniently. The only purpose in using a $\tau>0$ is that of avoiding initial $\delta$-functions. Notice that the total mass is 1 and $u_{0}$ is symmetric; hence, $u(x, t)$ will be symmetric in $x$ for every $t>0$.

We shall choose the $x_{i}$ inductively so as to have

$$
\bar{u}\left(0, t_{n} ; 1\right)-\max _{x} u\left(x, t_{n}\right) \geqslant C \varepsilon 2^{-n} t_{n}^{-1 /(m+1)}
$$

and

$$
\max _{x} \bar{v}\left(x, t_{n} ; 1\right)-\max _{x} v\left(x, t_{n}\right) \geqslant C \varepsilon 2^{-n} t^{-m /(m+1)}
$$

for some $C=C(m)>0$ so that (1.15), (1.16) follow. This is achieved by separating the points $x_{i}$ so much from each other that the different parts of $u$ (i.e. the disjoint components of the support of $u(\cdot, t))$ fail to join for times long enough: we begin by considering the solution $u^{0}(x, t)$ with initial condition $u_{0}^{0}(x)=\bar{u}(x, \tau ; 1-\varepsilon)$. Once we construct $u^{0}, u^{1}, \ldots, u^{n-1}$ for an integer $n \geqslant 1$ the inductive step is as follows: let $\tilde{u}^{n}$ be the solution with initial condition

$$
\tilde{u}_{0}^{n}(x)=\bar{u}\left(x-x_{n}, \tau ; \varepsilon / 2^{n+1}\right)+\bar{u}\left(x+x_{n}, \tau ; \varepsilon / 2^{n+1}\right)
$$

and let $u^{n}$ be the one with $u_{0}^{n}(x)=u_{0}^{n-1}(x)+\tilde{u}_{0}^{n}(x)$. We take $x_{n}$ large enough so as to have disjoint supports for $u^{n-1}(\cdot, t)$ and $\tilde{u}^{n}(\cdot, t)$ if $0<t<t_{n}$ (this is easy; use, for instance, the estimates on the support contained in [19] if you want to estimate $\left.x_{n}\right)$. Therefore, we have $u^{n}(x, t)=u^{n-1}(x, t)+\tilde{u}(x, t)$ in $Q_{t_{n}}$.

With this construction we have $u(x, t)=\lim u^{n}(x, t)$ in $Q$ as $n \rightarrow \infty$ and $\max _{x} u\left(x, t_{n}\right)=u^{n-1}\left(0, t_{n}\right), n \geqslant 1$. Therefore, using (1.9) and observing that the mass of $u^{n-1}$ is $1-\varepsilon 2^{-n}$ we have

$$
\begin{aligned}
t_{n}^{1 /(m+1)}\left(\bar{u}\left(0, t_{n} ; 1\right)-\max _{x} u(x, t)\right) & \geqslant t_{n}^{1 /(m+1)}\left(\bar{u}\left(0, t_{n} ; 1\right)-\bar{u}\left(0, t_{n}+\tau ; 1-\varepsilon 2^{-n}\right)\right) \\
& \geqslant d_{m}\left(1-\left(1-\varepsilon 2^{-n}\right)^{2 /(m+1)}\right) \geqslant \varepsilon C 2^{-n} .
\end{aligned}
$$

A similar argument gives (2.6). 
2.3. We discuss now the property of bounded variation. First we have the

Proof of Theorem 3. The inequality $v_{x} \leqslant((m+1) t)^{-1}$ allows us to control the positive variation of $v(\cdot, t)$ on any bounded interval. To control the negative variation we use the fact that, for any $x_{1}<x_{2}$ and $t>0, v\left(x_{2}, t\right)-v\left(x_{1}, t\right)=$ (positive variation of $v(\cdot, t)$ in $\left[x_{1}, x_{2}\right]$ ) - (negative variation of $v(\cdot, t)$ in $\left[x_{1}, x_{2}\right]$ ) and the estimates for $v$ in Theorem 1 .

Finally the inequality $\geqslant$ in (1.19) is a consequence of the fact that $\max v(x, t)$ and $-\min v(x, t)$ are equal or larger than $\max \bar{v}(x, t ; M)-\varepsilon$ for all large $t$ by $(1.11)$. The inequality $\leqslant$ in the same formula follows from (1.18) and the fact that $\left|\zeta_{i}(t)\right| / r(t) \rightarrow$ 1 as $t \rightarrow \infty,(1.5)$.

The existence of solutions with infinite variation is illustrated by the following

EXAMPLE. $u(x, t)$ is the solution of $(0.1)$ with initial data

$$
u_{0}(x)=\sum_{n=1}^{\infty} n^{-\alpha} \delta\left(x-x_{n}\right)
$$

where $1<\alpha<(m+1) /(m-1)$ so that $u_{0} \in L^{1}(\mathbf{R})$ but $\sum n^{-\alpha(m-1) /(m+1)}=\infty$. The sequence $\left\{x_{n}\right\}$ is positive, increasing and such that, for any positive $t>0$, $u(x, t)$ equals for large $x$ the sum $\sum \bar{u}\left(x-x_{n}, t ; n^{-\alpha}\right), n \geqslant n_{0}(t)$ (cf. the argument in Theorem 2). Now the variation of $v(\cdot, t)$ is bounded below by the sum of the variations of velocities corresponding to these $\bar{u}\left(x-x_{n}, t ; n^{-\alpha}\right)$, i.e. by

$$
\sum_{n \geqslant n_{0}(t)} c n^{-\alpha(m-1) /(m+1)} t^{-m /(m+1)},
$$

hence $\|v(\cdot, t)\| \|=\infty$ for every $t>0$. If you do not like the initial measures shift the origin of time to $t=\tau>0$ (as in Theorem 2).

3. The case $0<m<1$. As in the case $m>1$ there is a unique solution $u$ of the problem (0.1)-(0.3) but now $u \in C^{\infty}(Q)$ and $u>0$ in $Q$ (cf. [2]). This implies that at least when $u_{0}$ has compact support the velocity cannot be a bounded function in $Q$. To begin with the model solution $\bar{u}(x, t ; M)$ has an unbounded velocity: $\bar{v}(x, t ; M)=x /((m+1) t)$ in $Q$. For $m<1$ we may let $M \rightarrow \infty$ in $\bar{u}(x, t ; M)$ and obtain the solutions

$$
\begin{array}{ll}
\bar{u}_{\infty}(x, t)=\left(\frac{2 m(m+1) t}{(1-m) x^{2}}\right)^{1 /(m+1)} & \text { if } x \neq 0, t>0 \\
\bar{u}_{\infty,+}(x, t)=\bar{u}_{\infty}(x, t) & \text { if } x>0, t>0 \\
\bar{u}_{\infty,-}(x, t)=\bar{u}_{\infty}(x, t) & \text { if } x<0, t>0 .
\end{array}
$$

For all of them the velocity is given by the same $N$-wave in their respective domains of definition. We shall use the solutions (3.1) as suitable comparison functions in the sequel.

As for the convergence of a generic solution $u(x, t)$ towards its model $\bar{u}(x, t ; M)$, (1.3) is still valid (cf. [11]) and the estimate (1.9) $u(x, t) \leqslant \bar{u}(0, t ; M)$ is proved using the same argument as in $\$ 2$. It is proved in $N$ dimensions in [20]. The following result shows in what sense the velocities converge (we recall that now $v \in C^{\infty}(Q)$ for any solution). 
THEOREM 4. For every $\varepsilon, C>0$ there exists $T>0$ (depending also on the solution) such that if $t>T$ and $|x| \leqslant C M^{(m-1) /(m+1)} t^{1 /(m+1)}$ then

$$
t^{m /(m+1)}\left|v(x, t)-\frac{x}{(m+1) t}\right| \leqslant \varepsilon .
$$

Proof. Using invariant coordinates Theorem 4 is proved as a consequence of the inequality $v_{x} \leqslant((m+1) t)^{-1}$ (cf. [2]) and the convergence (1.3), arguing as in Theorems 1 and 3.

In some cases the convergence of $v$ towards $\bar{v}=x /((m+1) t)$ is not only local but also global in $x$. This happens for instance if $u_{0}$ has compact support as the following result shows.

THEOREM 5. Let $u$ be a solution of (0.1)-(0.3) and let $\alpha=$ infimum ( $\operatorname{support}\left(u_{0}\right)$ ) and $\beta=\operatorname{supremum}\left(\operatorname{support}\left(u_{0}\right)\right),-\infty \leqslant \alpha<\beta \leqslant \infty$. Then for every $t>0$ we have

$$
\begin{aligned}
& -\beta \leqslant(m+1) t v(x, t)-x \leqslant-\alpha, \\
& \lim _{x \rightarrow \infty}((m+1) t v(x, t)-x)=-\beta
\end{aligned}
$$

and

$$
\lim _{x \rightarrow x}((m+1) t v(x, t)-x)=-\alpha .
$$

Proof. (i) Case $m<1$. We begin by proving (3.4). Fix $t>0$. Since $(m+1) t v_{x} \leqslant 1$ there exists the limit

$$
\lim _{x \rightarrow \infty}((m+1) t v(x, t)-x)=-B,
$$

where $B$ can be either finite or $+\infty$. Therefore, for every $x$ we have $(m+1) t v(x, t)$ $\geqslant x-B$. Integrating this inequality and recalling that $v=(m /(1-m))\left(u^{m-1}\right)_{x}$ gives for $B<\infty$

$$
u(x, t) \leqslant\left(\frac{2 m(m+1) t}{(1-m)(x-B)^{2}}\right)^{1 /(m+1)}=\bar{u}_{\propto}(x-B, t)
$$

for every $x>B, t>0$.

We now need the following comparison result that we have introduced in [19] for $m>1$.

Shifting Comparison Lemma. Let $u, \hat{u}$ be solutions of $(0.1),(0.2)$ with respective initial data $u_{0}, \hat{u}_{0}$ satisfying $(0.3)$ and such that

$$
\int_{x}^{\infty} \hat{u}_{0}(s) d s \leqslant \int_{x}^{\infty} u_{0}(s) d s \quad \text { for every } x \in \mathbf{R} .
$$

Then for every $t>0$ we have

$$
\int_{x}^{\infty} \hat{u}(s, t) d s \leqslant \int_{x}^{\infty} u(s, t) d s \quad \text { for every } x \in \mathbf{R} .
$$

The proof of this version does not differ from the one in [19].

We are now in a position to prove that $B \geqslant \beta$ : choose any $\beta_{1}<\beta$, let $M_{1}=$ $\int_{\beta_{1}}^{B} u_{0}(x) d x$ and consider the solution $\hat{u}(x, t)$ with initial condition $\hat{u}_{0}(x)=$ $M_{1} \delta\left(x-\beta_{1}\right)$. It is clear that $\hat{u}_{0}$ and $u_{0}$ satisfy (3.8) so that, for every $t>0,(3.9)$ 
holds. Since $\hat{u}(x, t)=\bar{u}\left(x-\beta_{1}, t ; M_{1}\right)$ behaves as $x \rightarrow \infty$ like $\bar{u}_{\infty}\left(x-\beta_{1}, t\right)$, we conclude from (3.7) and (3.9) that $\beta_{1} \leqslant B$. Since $\beta_{1}$ is any number less than $\beta$ we conclude that $\beta \leqslant B$.

In the case $\beta<\infty$ we have yet to prove that $B \leqslant \beta$ to obtain (3.4). This is done like so: we consider the functions $u(x, t)$ and $\bar{u}_{\infty}(x-\beta, t)$. Since both are solutions of $(0.1)$ in the region $G_{+}=(\beta, \infty) \times(0, \infty)$, a standard comparison gives

$$
u(x, t) \leqslant \bar{u}_{\infty}(x-\beta, t) \text { in } G_{+} .
$$

Since (3.6) implies that $(m+1) t v(x, t) \leqslant x-B+\varepsilon$ for all sufficiently large $x$, integration of this inequality and comparison of the result with (3.10) imply that $B \leqslant \beta$.

The proof of (3.5) is analogous and (3.3) follows from (3.4), (3.5) and $(m+1) t v_{x}$ $\leqslant 1$.

(ii) Case $m=1$. We can use the integral representation of the solutions of the heat equation to prove our result. In fact, we have

$$
2 t \frac{u_{x}(x, t)}{u(x, t)}+x=\frac{\int e^{+x y / 2 t} y \varphi(y) d y}{\int e^{+x y / 2 t} \varphi(y) d y}
$$

where $\varphi(y)=u_{0}(y) \exp \left(-y^{2} /(4 t)\right)$.

COROllary 3.1. Let $m<1$. Under the assumptions of Theorem 5 , if $\alpha>-\infty$ we have for every fixed $t>0$

$$
u(x, t)=\left(\frac{2 m(m+1) t}{(1-m)(x-\alpha)^{2}}\right)^{1 /(1-m)}\left(1+o\left(\frac{1}{x}\right)\right) \text { as } x \rightarrow-\infty,
$$

and if $\beta<\infty$ we have

$$
u(x, t)=\left(\frac{2 m(m+1) t}{(1-m)(x-\beta)^{2}}\right)^{1 /(1-m)}\left(1+o\left(\frac{1}{x}\right)\right) \text { as } x \rightarrow \infty .
$$

We always have for every $t>0$

$$
\liminf _{|x| \rightarrow \infty} \frac{u(x, t)}{\bar{u}_{\infty}(x, t)} \geqslant 1 \text {. }
$$

Proof. (3.12), (3.13) follow by integration of (3.4), (3.5). Therefore, (3.14) is clear if $u_{0}$ has compact support. If not, let $\hat{u}_{0}$ be any function with compact support such that $0 \leqslant \hat{u}_{0} \leqslant u_{0}$ a.e. and apply the result to $\hat{u}$ recalling that $\hat{u} \leqslant u$ in $Q$.

REMARKS. (1) A striking feature of the asymptotic behaviour as $|x| \rightarrow \infty$ of the solutions of $(0.1)-(0.3)$ when $u_{0}$ has compact support and $m \leqslant 1$ is the fact that the first information about the initial data that is reflected in (3.12), (3.13) is not the mass (as in the case $m \geqslant 1$ ) but the endpoints of the support (cf. (3.12), (3.13)). This is a consequence of the fact that the diffusion coefficient has the form $c(u)=m u^{m-1}$ so that $c \uparrow \infty$ as $u \downarrow 0$.

(2) Using the above comparison arguments it follows easily from $u_{0}(x)=$ $o\left(|x|^{-2 /(1-m)}\right)$ as $|x| \rightarrow \infty$ that for every $t>0$

$$
\lim _{|x| \rightarrow \infty} \frac{u(x, t)}{\bar{u}_{\infty}(x, t)}=1 \text {. }
$$


Unbounded velocity occurs for a wide class of initial data as we show next.

THEOREM 6. Let $m<1$ and let $u_{0} \in L^{1}(\mathbf{R}), u_{0} \geqslant 0, u_{0} \neq 0$ and $u_{0}(x)=$ $o\left(|x|^{-1 / 1-m)}\right)$ as $x \rightarrow \infty$ (respectively $\left.x \rightarrow-\infty\right)$. Then $v(\cdot, t)$ is not bounded above (resp. below) for any $t>0$. The result holds for $m=1$ if the last assumption is changed into $\lim \lg \left(u_{0}(x)\right) /|x|=-\infty$ as $x \rightarrow \infty$ (resp. $\left.x \rightarrow-\infty\right)$.

Proof. Let $m<1$. If, at a certain time $t>0, v(\cdot, t)$ is bounded above, say by $C>0$, then integrating $v=--m u^{m-2} u_{x}$ gives for $x>0$ the estimate

$$
u(x, t) \geqslant\left(c_{1}+c_{2} x\right)^{-1 /(1-m)}
$$

for some constants $c_{1}, c_{2}>0$ (depending on $C$ and $m$ ). However, we are going to prove next that if $u_{0}(x)=o\left(|x|^{-1 /(1-m)}\right)$ as $x \rightarrow \infty$ then the same estimate must be true for every $t>0$, thus contradicting (3.16). In fact, the function

$$
\hat{u}(x, t)=\left(\frac{1-m}{m c\left(x-c t-x_{0}\right)}\right)^{1 /(1-m)},
$$

where $c>0$ and $x_{0}$ are constants, is a solution of $(0.1)$ in the domain $G=\{(x, t)$ : $\left.x>c t+x_{0}\right\}$; since $\hat{v}(x, t)=c, \hat{u}$ is called a "constant-velocity front". It follows from a standard comparison in $G$ that for any $c>0$ we can find an $x_{0}$ (possibly large) such that $\hat{u}(x, t) \geqslant u(x, t)$ in $G$. Since

$$
\hat{u}(x, t) x^{1 /(1-m)} \rightarrow(m c /(1-m))^{-1 /(1-m)} \text { as } x \rightarrow \infty
$$

and $c$ is arbitrary, we have $u(x, t)=o\left(|x|^{-1 /(1-m)}\right)$.

The argument for the lower bound is the same. For $m=1$ the constant velocity front is defined in $Q$ by

$$
\hat{u}(x, t)=c_{1} \exp (c(c t-x)) .
$$

The rest of the argument is easily adapted.

Remarks. (3) Using the Shifting Comparison Lemma the condition $u_{0}(x)=$ $o\left(|x|^{-1 /(1-m)}\right)$ can be replaced by $\int_{x}^{\infty} u_{0}(s) d s=o\left(|x|^{-m /(1-m)}\right)$ as $x \rightarrow \infty$; similarly as $x \rightarrow-\infty$.

In the proof of Theorem 6 we have constructed an explicit solution of $(0.1)$, $0<m<1$, in an infinite subdomain of $Q$ having bounded velocity. This solution behaves at infinity like $O\left(x^{-1 /(1-m)}\right)$. In the next section a class of solutions in all of $Q$, having bounded velocity and therefore behaving as $|x| \rightarrow \infty$ as $\geqslant C|x|^{-1 /(1-m)}$, is exhibited. Thus, in particular, the exponent in Theorem 6 is sharp. A similar comment applies to the case $m=1$.

4. A class of solutions with bounded velocity. In this section we consider a more general problem consisting of the equation

$$
u_{t}=(\phi(u))_{x x} \text { in } Q=\mathbf{R} \times(0, \infty)
$$

where $\phi$ is a continuous, nondecreasing function $[0, \infty) \rightarrow[0, \infty)$ with $\phi(0)=0$ and the initial data

$$
u(x, 0)=u_{0}(x)
$$


where $u_{0} \in L^{1}(\mathbf{R}), u_{0} \geqslant 0$ and $\int u_{0}(x) d x=M>0$. Using the terminology of the preceding sections we think of $u$ as a density and define the pressure as $p=\psi(u)$ where

$$
\psi(u)=\int_{1}^{u} \frac{d \phi(s)}{s}, \quad 0<u<\infty
$$

(the second member is well defined as a Riemann-Stieltjes integral). $\psi$ is a continuous function in $(0, \infty)$. We remark that the origin of pressures is unimportant. Conversely, $u$ is well defined by $p, u=\psi^{-1}(p)$, whenever $\phi$ is strictly increasing. The balance argument (0.4) implies that the velocity $v$ satisfies $v=-p_{x}$.

A solution $u \in C\left([0, \infty): L^{1}(\mathbf{R})\right)$ of (4.1), (4.2) can be obtained, for instance, by discretization in time. The maps $S_{t}: u_{0} \rightarrow u(\cdot, t)$ form a semigroup of $L^{1}$-contractions and if $u_{0} \in L^{\infty}(\mathbf{R})$ then $u(\cdot, t) \in L^{\infty}(\mathbf{R})$ and it is the unique solution of (4.1) or $\mathscr{D}^{\prime}(Q)$ that satisfies the initial condition (4.2). Moreover, the solutions depend continuously on $\phi$ : if $\phi_{n}$ is a sequence of functions as above and $\phi_{n}(r) \rightarrow \phi(r)$ for every $r \in \mathbf{R}$ then (with obvious notation) $u_{\Phi_{n}} \rightarrow u_{\varphi}$ in $C\left([0, \infty): L^{1}(\mathbf{R})\right.$ ). We refer to [4] for this material. For uniqueness see [7].

Our result is the following

THEOREM 7. Assume that $u(x, t)$ is the solution of (4.1), (4.2) as above. If $\psi\left(u_{0}\right)$ is a Lipschitz-continuous function with constant $L_{0}$ then, for every $t>0, \psi(u(\cdot, t))$ is also Lipschitz-continuous with constant $L(t) \leqslant L_{0}$.

Remarks. (1) A continuous function $u_{0}$ as in the theorem can vanish only if

$$
\int_{0}^{1} \frac{d \phi(s)}{s}<\infty
$$

If $\phi(s)=s^{m}, m>0$, this happens if and only if $m>1$. In [12] Kalashnikov introduced the change of variables (4.3) under condition (4.4) and proved that the velocity of any solution of (4.1), (4.2) is then bounded for $t \geqslant \tau>0$ (he uses for convenience additional smoothness hypotheses on $\phi$ and $u_{0}$ that are inessential and can be eliminated with the arguments that we shall expound). As a consequence, under condition (4.4) solutions of (4.1), (4.2), where $u_{0}$ has compact support, are compactly supported in $x$ for every $t>0$.

(2) If the integral (4.3) is divergent as $u \rightarrow \infty\left(m \geqslant 1\right.$ if $\left.\phi(s)=s^{m}\right)$ then every function $u_{0}$ as above is bounded, and then $u(x, t)$ is the unique solution of (4.1), (4.2).

(3) The theorem is true for any solution of (4.1) that can be conveniently approximated by bounded solutions. Thus, for instance, if $\phi(s)=s^{m}, m>1$, Bénilan, Crandall and Pierre [5] have constructed solutions for an optimal class of initial data $u_{0}$; in particular, under the assumptions $u_{0} \geqslant 0, u_{0}^{m-1}$ is Lipschitzcontinuous.

(4) Solutions with constant velocity (that generalize those shown in $\S 3$ for $\phi(s)=$ $\left.s^{m}\right)$ can be constructed for (4.1). Let us fix the velocity $c>0$. The front is defined in the set $G=\{(x, t) \in Q: \psi(0)<c(c t-x)<\psi(\infty)\}$ in terms of the pressure by

$$
p(x, t)=c(c t-x) \text {. }
$$


If $\phi$ is strictly increasing this defines a solution $u$ of $(4.1)$ in $G$. The front is a solution in $Q$ if the integral (4.3) diverges at 0 and at $\infty\left(m=1\right.$ if $\left.\phi(s)=s^{m}\right)$. In no case does it belong to $L^{1}(\mathbf{R})$ as a function of $x$ for any fixed $t$.

Proof of TheOREM 7. It consists of two parts. First we perform a classical calculation. Then we adapt this calculation to our solutions by an approximation process. The first part is contained in the following

LEMMA 4.1. Let $u(x, t)$ be a classical solution of the equation $u_{t}=(\phi(u))_{x x}$ in $a$ cylindrical domain $Q_{T, l}=\{(x, t):|x| \leqslant l, 0<t<T\}, u \in C^{1}\left(\bar{Q}_{T, l}\right) \cap C^{3}\left(Q_{T, l}\right)$, where $\phi$ is an increasing function, $\phi \in C^{1}[0, \infty) \cap C^{2}(0, \infty), \phi^{\prime}(s)>0$ if $s>0$. Let $u>0$ in $Q_{T, l}$ and let $\partial u / \partial x=0$ on the lateral boundary $S_{T, l}=\{(x, t):|x|=l$, $0 \leqslant t \leqslant T\}$. Then the maximum and the minimum of $v(x, t)=-\phi^{\prime}(u) u^{-1} u_{x}$ are attained at $t=0$.

Proof. Performing the change of variable $u \rightarrow p=\psi(u)$ given by (4.3) we obtain the equation, for $p$,

$$
p_{t}=\sigma(p) p_{x x}+p_{x}^{2}
$$

where $\sigma(p)=\varphi^{\prime}(u)$. Differentiating with respect to $x$ and setting $v=-p_{x}$ gives

$$
v_{t}=\sigma(p) v_{x x}-\left[\sigma^{\prime}(p)+2\right] v_{x} v \text {. }
$$

Take now $\varepsilon>0$ and let $w=v \exp (-\varepsilon t)$. If the maximum of $w$ is positive and is attained in $Q$ we have at this point $w_{t}=v_{t} \exp (-\varepsilon t)-\varepsilon v \exp (-\varepsilon t) \geqslant 0, \quad w_{x x}=$ $v_{x x} \exp (-\varepsilon t) \leqslant 0, w_{x}=v_{x} \exp (-\varepsilon t)=0$. All this together contradicts (4.7). Hence a positive maximum must be attained on the parabolic boundary and, since $w=0$ on its lateral part, it must be attained at $t=0$. Now let $\varepsilon \rightarrow 0$ to get the same conclusion for $v$. A similar argument applies to the minimum.

Second Part of Proof of Theorem 7. Since Lemma 4.1 does not apply directly to our solutions we perform an approximation process in several steps.

(i) We assume also that $u_{0}$ is bounded and $\phi \in C^{1}[0, \infty) \cap C^{5}(0, \infty), \phi^{\prime}(s)>0$ for every $s>0$ : According to Oleinik et al. [17, Theorem 2] the solution can be obtained as the limit of positive solutions $u_{n}$ defined in an expanding sequence of cylinders $Q_{n}$. These approximate solutions satisfy the assumptions of Lemma 4.1 except the boundary condition; but we can modify the construction using [17, Theorem 10] to fulfill this condition. Moreover, we can choose the initial functions $u_{n}^{0}$ such that the Lipschitz constant of $\psi\left(u_{n}^{0}\right)$ does not exceed $L_{0}$. Applying Lemma 4.1 to the approximate solutions we get $\left|v_{n}(x, t)\right| \leqslant L_{0}$ in $Q_{n}$, hence $|v(x, t)| \leqslant L_{0}$.

(ii) $u_{0}$ is bounded, $\phi^{\prime}(s)>0$ if $s>0$ : We approximate $\phi$ by an increasing sequence $\phi_{n}$ as in (i) such that $\psi_{n}\left(u_{0}\right)$ has Lipschitz constant at most $L_{0}\left(\psi_{n}\right.$ is defined by replacing $\phi$ by $\phi_{n}$ in (4.3)). Then $\phi_{n} \rightarrow \phi$ in compact subsets of $[0, \infty)$ by Dini's theorem. Since

$$
\psi_{n}(s)=\frac{\phi_{n}(s)}{s}-\phi_{n}(1)-\int_{1}^{u} \frac{\phi_{n}(s)}{s^{2}} d s,
$$

also $\psi_{n} \rightarrow \psi$ uniformly on compact subsets of $(0, \infty)$. 
Let $u_{n}$ be the solution of $u_{t}=\left(\phi_{n}(u)\right)_{x x}, u(x, 0)=u_{0}$. It follows from [4] that $u_{n} \rightarrow u$ in $C\left([0, \infty): L^{1}(\mathbf{R})\right)$ and, after passing to a subsequence, a.e. in $Q$. Also $v_{n}(x, t)=-\left(\psi_{n}\left(u_{n}(x, t)\right)\right)_{x}$ is bounded in $Q$ by $L_{0}$. Now we estimate the Lipschitz constant of $p(\cdot, t)$ for a $t>0$. We have

$$
\begin{aligned}
p\left(x_{1}, t\right)- & p\left(x_{2}, t\right)=\left[\psi\left(u\left(x_{1}, t\right)\right)-\psi\left(u_{n}\left(x_{1}, t\right)\right)\right] \\
& +\left[\psi\left(u_{n}\left(x_{2}, t\right)\right)-\psi\left(u\left(x_{2}, t\right)\right)\right]+\left[\psi\left(u_{n}\left(x_{1}, t\right)\right)-\psi_{n}\left(u_{n}\left(x_{1}, t\right)\right)\right] \\
& +\left[\psi_{n}\left(u_{n}\left(x_{2}, t\right)\right)-\psi\left(u_{n}\left(x_{2}, t\right)\right)\right]+\left[\psi_{n}\left(u_{n}\left(x_{1}, t\right)\right)-\psi_{n}\left(u_{n}\left(x_{2}, t\right)\right)\right] .
\end{aligned}
$$

Since $v_{n}(x, t)$ is bounded by $L_{0}$ the last difference is at most $L_{0}\left|x_{2}-x_{1}\right|$. The other differences in the second member of (4.9) can be made arbitrarily small as $n \rightarrow \infty$ for almost every $x_{1}, x_{2}$ such that $u\left(x_{1}, t\right), u\left(x_{2}, t\right)$ do not vanish: the two first parentheses are small because $u_{n} \rightarrow u$ a.e., hence $\psi\left(u_{n}\right) \rightarrow \psi(u)$ a.e. For the following two parentheses we remark that $\left|u_{n}(x, t)\right| \leqslant \sup \left|u_{0}(x)\right|=N<\infty$ and that $\psi_{n} \rightarrow \psi$ uniformly on $[0, N]$. Letting $n \rightarrow \infty$ we obtain for a.e. $x_{1}, x_{2}$ such that $u\left(x_{1}, t\right), u\left(x_{2}, t\right) \neq 0$ that $\left|p\left(x_{1}, t\right)-p\left(x_{2}, t\right)\right| \leqslant L_{0}\left|x_{1}-x_{2}\right|$. The conclusion follows.

(iii) $u_{0}$ is bounded. We construct a decreasing sequence of functions $\phi_{n}$ as in the hypotheses of (ii) and such that $\psi_{n}\left(u_{0}\right) \leqslant L_{0}+1 / n$ and follow the approximation argument of (ii).

(iv) If $u_{0}$ is not bounded, take $u_{n}^{0}=\min \left(u_{0}(x), n\right)$ and apply the theorem to the solutions $u_{n}=S_{t} u_{n}^{0}$. Then let $n \rightarrow \infty$.

REMARKS. (5) Shifting the origin of time we conclude from Theorem 7 that $L(t)$ is a nonincreasing function of $t, t \geqslant 0$. Returning to $(0.1): u_{t}=\left(u^{m}\right)_{x x}$, it follows from Theorem 1 that, whenever $m>1, L(t) \rightarrow 0$ as $t \rightarrow \infty$. This is not the case for every solution of $(0.1),(0.2)$ if $m \leqslant 1$, even if the initial velocity is bounded. In fact, if $u_{0}$ is a nonnegative function of the class $C^{1}$ such that

$$
\lim _{|x| \rightarrow \infty}\left|v_{0}(x)\right| \equiv \lim _{|x| \rightarrow \infty} \frac{m\left|u_{0}^{\prime}(x)\right|}{u_{0}(x)^{2-m}}>0
$$

then $\lim L(t)>0$ as $t \rightarrow \infty$. This result can be proved using the techniques of $\S 3$.

\section{REFERENCES}

1. D. C. Aronson, Regularity properties of flows through porous media, SIAM J. Appl. Math. 17 (1969), 461-467.

2. D. C. Aronson and P. Bénilan, Régularité des solutions de l'équation des milieux poreux dans $\mathbf{R}^{n}$. C. R. Acad. Sci. Paris Sér. A-B 288 (1979), 103-105.

3. G. I. Barenblatt, On some unsteady motions of a liquid or a gas in a porous medium. Prikl. Mat. Mekh. 16 (1952), 67-78. (Russian)

4. P. Bénilan and M. G. Crandall, The continuous dependence on $\phi$ of the solutions of $u_{1}-\Delta \phi(u)=0$, Indiana Univ. Math. J. 30 (1981), 161-177.

5. P. Bénilan, M. G. Crandall and M. Pierre, Solutions of the porous media equation in $\mathbf{R}^{\prime \prime}$ under optimal conditions on initial values, Indiana Univ. Math. J. 33 (1984), 51-87.

6. J. G. Berryman and C. J. Holland, Stability of the separable solution for fast diffusion, Arch. Rational Mech. Anal. 74 (1980), 379-388.

7. H. Brezis and M. G. Crandall, Uniqueness of solutions of the initial-value problem for $u_{t}-\Delta \varphi(u)=0$. J. Math. Pures Appl. 58 (1981), 162-177. 
8. E. D. Conway and E. Hopf, Hamilton's theory and generalized solutions of the Hamilton-Jacobi equation, J. Math. Mech. 13 (1964), 939-986.

9. C. M. Dafermos, Asymptotic behaviour of solutions of hyperbolic balance laws, Bifurcation Phenomena in Mathematical Physics and Related Topics, Proceedings NATO Adv. Study Inst., Cargèse, Corsica, 1979. pp. 521-533.

10. R. T. Di Perna, Decay and asymptotic behaviour of solutions to nonlinear hyperbolic systems of conservation laws, Indiana Univ. Math. J. 24 (1975), 1047-1071.

11. A. Friedman and S. Kamin. The asymptotic behaviour of a gas in an n-dimensional porous medium. Trans. Amer. Math. Soc. 262 (1980), 551-563.

12. A. S. Kalashnikov, On the differential properties of generalized solutions of equations of the nonsteady-state filtration type, Vestnik Moskov Univ. Ser. I Mat. Mekh. 29 (1974), 62-68 (translation 48-53).

13. S. Kamin, The asymptotic behaviour of the solution of the filtration equation, Israel J. Math. 14 (1973), 76-78.

14. P. Lax, Hyperbolic systems of conservation laws. II, Comm. Pure Appl. Math. 10 (1957), 537-566.

15. T.-P. Liu, Decay to $N$-waves of solutions of general systems of nonlinear hyperbolic conseriation laws, Comm. Pure Appl. Math. 30 (1977), 585-610.

16. T.-P. Liu and M. Pierre, Source-solutions and asymptotic behavior in conservation laws, MRC Technical Summary Report \#2318, 1982; J. Differential Equations 51 (1984), 419-441.

17. O. A. Oleinik, A. S. Kalashnikov and C. Yui-Lin, The Cauchy problem and boundary problems for equations of the type of nonstationary filtration. Izv. Akad. Nauk Armyan SSR Ser. Mat. 22 (1958), 667-704. (Russian)

18. L. A. Peletier, The porous media equation, Applications of Nonlinear Analysis in the Physical Sciences (H. Amann et al., eds.), Pitman, London, 1981, pp. 229-241.

19. J. L. Vazquez, Asymptotic behaviour and propagation properties of the one-dimensional flow of gas in a porous medium, Trans. Amer. Math. Soc. 277 (1983), 507-527.

20. Symetrisation pour $u_{t}=\Delta \varphi(u)$ et applications, C. R. Acad. Sci. Paris Sér. I 295 (1982), $71-74$.

21. The interfaces of one-dimensional flows in porous media, Trans. Amer. Math. Soc. (to appear).

SChool of Mathematics, University of Minnesota, Minneapolis, Minnesota 55455

Current address: División de Matemáticas, Universidad Autónoma, Madrid 34, Spain 